\title{
TECNOLOGIAS ASSISTIVAS PARA AUXILIAR DEFICIENTES VISUAIS NA CRIAÇÃO E LEITURA DE CONTEÚDOS GRÁFICOS: UMA REVISÃO SISTEMÁTICA DA LITERATURA
}

\author{
Leonardo Zamprogno ${ }^{1}$, Bruno Merlin ${ }^{1}$, João Ferreira ${ }^{2}$, Heleno Fülber ${ }^{1}$, Allan \\ Veras $^{1}$ \\ ${ }^{1}$ Programa de Pós-Graduação em Computação aplicada - Universidade Federal do Pará \\ (UFPA) - Campus Tucuruí - Tucuruí - PA - Brasil \\ ${ }^{2}$ Instituto Federal de Educação, Ciência e Tecnologia do Pará (IFPA) - Campus Tucuruí \\ - Tucuruí - PA - Brasil \\ \{leonardozani07, bruno.merlin, fulber, allanverasce\}@gmail.com, \\ joao.elias@yahoo.com.br
}

\begin{abstract}
Graphic contents are key resources to display certain informations. People with visual impairments do not have an easy access to this resource because of their impairment. Assistive technologies have been developed to help them access graphic content. This article presents a systematic review of the literature that aims at conducting a survey about assistive technologies developed to assist visual impaired people in reading and interpreting graphic contents. The review followed the proposed guidelines for this kind of research and, as a result, presents 51 studies about technologies that help visual impairment peoples in the creation and reading of graphic contents. We observed that, in this field of research, it is possible to improve technologies that will help to reduce the limitations found by the visually impaired individuals in accessing graphic contents and thus to reach higher levels of accessibility.
\end{abstract}

Resumo. $O$ acesso à conteúdos gráficos é um recurso fundamental para a comunicação, ao passo que, algumas informações são melhor transmitidas em formato de imagem, mapa, gráfico, etc., porém, pessoas com deficiência visual não têm acesso facilitado a este recurso por conta de sua deficiência. Tecnologias assistivas foram desenvolvidas para facilitar o acesso a conteúdo gráficos para os deficientes visuais. Este artigo apresenta uma revisão sistemática da literatura que busca realizar um levantamento das tecnologias assistivas para auxiliar deficientes na leitura e interpretação de conteúdos gráficos. A revisão seguiu as diretrizes propostas para esse tipo de pesquisa e os resultados apresentam 51 estudos sobre tecnologias que auxiliam na criação, leitura e interpretação de conteúdos gráficos. Observamos que neste campo de investigação é possível aprimorar tecnologias que ajudarão a diminuir as limitações encontradas pelos deficientes visuais em acessar conteúdos gráficos e assim poder alcançar níveis mais elevados de acessibilidade.

\section{Introdução}

Os conteúdos gráficos, em um sentido amplo que inclui também imagens e mapas, são eficazes em transmitir quantidade de informações em um pequeno espaço, bem como, melhor organizar uma série de dados e informações para torná-los facilmente compreensíveis. Eles são amplamente utilizados no processo de ensino sem distinção 
sobre o nível de escolaridade. A compreensão global e rápida de conteúdos gráficos é naturalmente permitida graças ao sentido da visão. Ao passo que alunos com deficiência visual (DV) não têm acesso tão fácil a este tipo de conteúdo, a utilização de outros subsistemas sensoriais, como audição e háptico (tato e cinestésico), principalmente, podem auxiliar na interpretação dos conteúdos gráficos.

Toda a legislação brasileira sobre inclusão das pessoas com deficiência, é relativamente ambiciosa. No entanto, muitas dificuldades são encontradas por parte das instituições no que tange, principalmente, ao orçamento e à capacitação de pessoal para receber alunos com deficiência na perspectiva de poder prestar-lhes todo o suporte necessário para o bom desenvolvimento educacional. Silva, Turatto e Machado (2002) afirmam que "o que se observa é a existência de uma contradição entre o discurso e a realidade, pois o deficiente visual, que depende de recursos públicos para sua educação enfrenta muitas barreiras e desafios".

As tecnologias assistivas (TA), segundo CAT (2009), "são recursos que potencializam as habilidades funcionais das pessoas com deficiência" e, portanto, podem ser uma forma de suprir as lacunas na formação dos docentes e a falta de material especializado. No que tange à apresentação de conteúdos gráficos, são vários desafios a enfrentar nos quais as TA poderiam ajudar como, principalmente, a elaboração de gráficos táteis corretamente elaborados, e tornar a leitura dos gráficos táteis pelos deficientes visuais autônoma.

Assim, este trabalho consiste em uma revisão sistemática da literatura (RSL), visando a estabelecer o estado da arte. Foi feita a análise e a categorização da literatura publicada sobre tecnologias assistivas para auxiliar deficientes visuais a acessar conteúdos gráficos.

Os próximos tópicos do presente artigo estão estruturados da seguinte maneira: a Seção 2 apresenta a metodologia utilizada na RSL, a questão de pesquisa, bem como suas sub-questões que ajudam a embasá-la, assim como é apresentado o processo de seleção dos estudos e condução; na Seção 3 é feita a análise dos dados extraídos dos estudos; na Seção 4 são dadas as respostas para as questões de pesquisa; na Seção 5 estão a conclusão do trabalho e as considerações finais.

\section{Método}

A metodologia adotada para este trabalho foi desenvolvida por Kitchenham e Charters (2007) a qual define que uma RSL deve possuir três fases: planejamento, condução e documentação da revisão. $\mathrm{Na}$ fase de planejamento foram definidas as questões de pesquisa, string de busca, definição das fontes de pesquisa e critérios de seleção (inclusão e exclusão) dos artigos. Após o planejamento seguiu-se a condução da revisão, documentação e apresentação dos resultados.

\subsection{Planejamento}

Esta RSL tem como questão de pesquisa: Quais tecnologias assistivas estão sendo usadas para auxiliar alunos com deficiência visual a ler e interpretar conteúdos gráficos?

Para ajudar a responder esta questão foram definidas algumas sub-questões apresentadas conforme a seguir: QP1- Qual é o número de participantes nos testes dos estudos que estão sendo realizados pelos pesquisadores? QP2 - Qual o nível de deficiência visual dos participantes dos estudos? QP3 - Para quais os níveis de escolaridade que estão sendo destinados os estudos? QP4 - Quais estratégias estão sendo usadas por 
pesquisadores que investigam formas de auxiliar a exploração de gráficos táteis no ensino para deficientes visuais? QP5 - Os estudos reportados indicam que o uso da tecnologia assistiva, desenvolvida ou analisada, é eficaz para auxiliar na leitura e interpretação de conteúdos gráficos? QP6 - Para quais tipos de conteúdo as soluções foram aplicadas? QP7 - Em quais países estão sendo realizados os estudos?

Os dados foram reunidos a partir da pesquisa em de quatro bases bibliográficas no período de 2006 a 2019. As bases consultadas foram: Web of Science, Scopus, ACM Digital Library e portal de periódicos da CAPES. Optou-se por selecionar fontes mais por referenciarem documentos de diversos editores como, como IEEE, Spring e Science Direct entre outras. Nas quatro bases, foram realizadas buscas com a string: ("assistive technolog*" OR "accessibilit*") AND ("tactile graphics" OR "tactile images" OR "tactile charts" OR "tactile diagrams" OR "accessible diagrams" OR "accessible charts" OR "accessible graphics").

Como critérios de inclusão dos estudos adotou-se selecionar (a) artigos com texto completo do estudo disponível em formato eletrônico; (b) trabalhos relativos a tecnologias assistivas para auxiliar deficientes visuais a ler e interpretar conteúdos gráficos; (c) estudos devem estar no idioma Inglês ou Português; (d) trabalhos que se referirem a mesma tecnologia considerar-se-á o mais atual.

Como critérios de exclusão adotou-se os seguintes: (a) estudos que fogem da área de pesquisa; (b) estudos que não estejam disponíveis online pela rede CAFe (Comunidade Acadêmica Federada da RNP) ou que não obtivemos acesso após contato com os autores; (c) estudos escritos em outras línguas; (d) resumos.

\subsection{Condução}

Foram encontrados na pesquisa bibliográfica um total de 382 artigos (CAPES - 162, ACM - 98, Web of Science - 42 e Scopus - 80). A relação com todos os artigos pode ser acessada através do link https://graficosdeficientesvisuais.blogspot.com/. Inicialmente foi feita uma análise dos títulos, resumos e palavras chaves para verificar se os estudos tinham relação com o objeto de pesquisa desta RSL. Após esta etapa foram retirados os estudos que não atendiam a este critério, do total, 241 artigos foram excluídos, restando 141 estudos. Com aplicação dos critérios de inclusão e exclusão descritos nos parágrafos anteriores, restaram 66 artigos (CAPES - 12, ACM - 27, Web of Science - 10 e Scopus 17). Em seguida, foram excluídos os estudos duplicados e correlacionados chegando ao resultado final deste levantamento somando um total de 51 estudos.

Após a leitura destes estudos pode-se identificar 51 soluções para criação e apresentação de conteúdos gráficos para deficientes visuais (11 para criação e 40 para apresentação). Devido à quantidade de soluções encontradas não foi possível apresentálas no corpo deste artigo, porém, as soluções, assim como as referências podem ser acessadas no link https://graficosdeficientesvisuais.blogspot.com/.

\section{Tecnologias assistivas para conteúdos gráficos}

As ferramentas para criação de conteúdos gráficos foram classificadas de acordo com a forma de criação do gráfico tátil, sendo: uma forma automática ou semiautomática ou uma forma manual. As tecnologias para leitura de gráficos táteis encontradas nesta RSL foram classificadas de acordo com as respostas sensoriais utilizadas para restituir a informação do gráfico: feedback auditivo, tátil, cinestésico e híbrido (ou multimodal). 


\subsection{Ferramentas para criação de conteúdos gráficos}

\subsubsection{Método de criação manual}

Uma das maneiras para pessoas, com deficiência visual, acessarem conteúdos gráficos é utilizando gráficos táteis. Anteriormente criar um gráfico tátil era complexo havendo a necessidade de transcritores profissionais para diagramar os gráficos táteis de maneira manual. Atualmente é possível encontrar trabalhos realizados de forma manual, porém com uso de ferramentas gráficas como Corel Draw e Inkscape, bem como soluções mais específicas como Graph Builder [Mcgookin e Brewster, 2006] e GraAuthor [Goncu e Marriott, 2015a].

\subsubsection{Método de criação semiautomática ou automáticas}

Existem, também, soluções que auxiliam na criação de gráficos táteis de maneira automática ou semiautomática. Algumas soluções geram gráficos por meio da inclusão de dados manualmente: Tactile Charts Generation Tools [Goncu e Marriott, 2008], VizTouch [Brown; Hurst, 2012] e GraCALC [Goncu e Marriott, 2015b]. Outras soluções podem gerar gráficos automaticamente por meio de processamento de imagens: TGA [Jayant et al., 2007] e Krufka [Krufka e Barner, 2005].

\subsection{Ferramentas para leitura de gráficos táteis}

\subsubsection{Feedback auditivo}

Os dispositivos com feedback auditivo fornecem informações sobre o gráfico de maneira sonora, utilizando voz sintetizada ou apenas pistas sonoras para orientar leitura e a navegação dentro do gráfico. Dentre estas soluções pode-se citar TGV [Baker et al., 2016], TGH [Fusco e Morash, 2015] e TacTILE [He et al., 2017].

\subsubsection{Feedback háptico tátil}

Os dispositivos com feedback háptico tátil tem como principal responsável, pelas respostas aos estímulos os sensores cutâneos que estão na pele e sentem o toque, alguns destes dispositivos utilizam esta estratégia de feedback por meio de atuadores mecânicos: FluxMarker [Suzuki et al., 2017], Displays Táteis [Bornchein, Bornchein e Weber, 2018] e VTD [Maucher, Schemmel e Meier, 2000]. Existem dispositivos que fornecem este tipo de feedback por meio de eletro vibrações como o Tesla Touch [Bau et al., 2010].

\subsubsection{Feedback háptico cinestésico}

Os dispositivos de feedback cinestésicos aplicam forças que movimentam parte do corpo do deficiente visual, ou seja, são aplicados aos músculos e articulações. Assim como dispositivos de feedback háptico tátil a maioria dos dispositivos desenvolvidos com essa estratégia se apoiam em hardware específico, pode-se citar: Senseable Phantom e Mouse de força WingMan [Yu e Brewster, 2002].

\subsubsection{Feedback hibrido}

Visando alcançar uma resposta sensorial maior e proporcionar mais facilidade na interpretação dos dados gráficos a serem exibidos, alguns dispositivos combinam duas ou mais formas de feedback, isto também é conhecido como multimodalidade de resposta sensorial. Alguns exemplos destes dispositivos e que utilizam hardware específico são: Talking Tactile Tablet (TTT) [Landau e Erin, 2007], Talking Tactile Pen (TTP) [Landau et al., 2008] e IVEO Touchpad [Gardner e Bulatov, 2006]. Já para os que utilizam hardware comum tem-se: GraVVITAS e GraVIEWER [Goncu e Marriott, 2011, 2015], Figure Perceiving Tool [Costa et al., 2015] e Vibro Audio-Interface [Giudice et al., 2012]. 


\section{Resultados e discussões}

Nessa Seção serão apresentadas as respostas para as questões definidas e discutidos os resultados. Os estudos levados em consideração para responder às questões de pesquisa também podem ser encontrados na página https://graficosdeficientesvisuais.blogspot.com/.

Para responder à questão 1: Qual é o número de participantes nos testes dos estudos que estão sendo realizados pelos pesquisadores? É importante ressaltar que os testes de uma solução podem ajudar a definir a efetividade da mesma, por isso a quantidade de participantes no estudo foi um dado importante levado em consideração por esta RSL.

Acerca da questão de pesquisa observa-se nos estudos selecionados por esta RSL que o número de participantes nas pesquisas não é significativamente alto devido à dificuldade de encontrar grupos de pessoas portadoras de deficiência visual disponíveis, ainda menos apresentando características homogêneas de deficiência, para participar de estudos e pesquisas, como citado em Goncu e Marriott (2011) e Petit et al. (2008). Consequentemente o número de participantes dos estudos, normalmente, é muito pequeno.

Dentre as publicações, base deste trabalho, apenas 19 estudos afirmam usar apenas participantes com algum tipo de deficiência visual. Somando um total de 164 participantes, resulta uma média de 9 por estudo, aproximadamente. Cabe ressaltar que estudos com um número maior de participantes se deve ao fato de incluírem, em sua maioria, videntes com os olhos vendados. Realizando o mesmo levantamento, porém levando em consideração os estudos que também utilizam este artifício, apenas 41 citaram a quantidade de participantes, resultando no total de 600 participantes e uma média de 15 por estudo, aproximadamente. Isto comprova que estudos desta natureza normalmente, são testados com poucos participantes e menos ainda quando testados exclusivamente com DV.
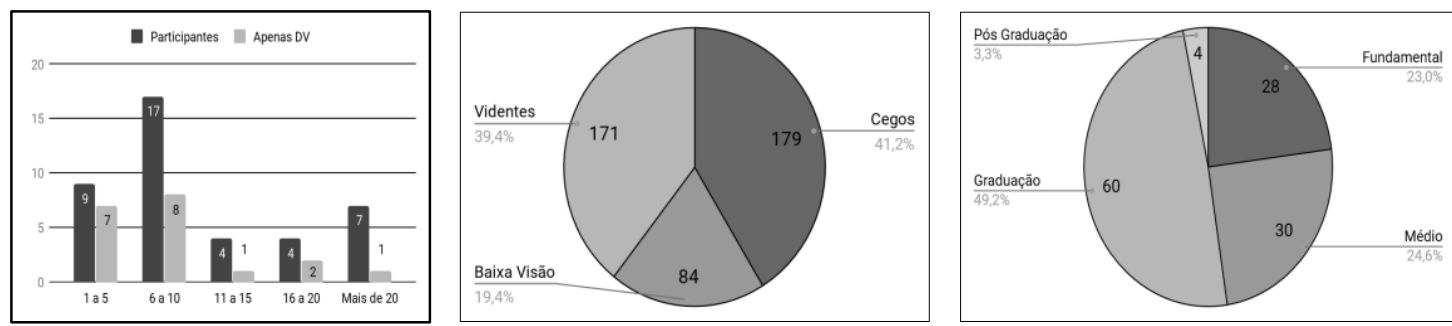

Figura 1. À esquerda, gráficos das quantidades de participantes por estudo $\mathrm{x}$ apenas DVs. No centro, quantidade de qualquer tipo de participante dos estudos. À direita nível de escolaridade dos participantes.

Para responder à questão 2: Qual o nível de deficiência visual dos participantes dos estudos? Os níveis de deficiência, levados em consideração pelos estudos, são os mesmos descritos no decreto $n^{\circ} 3.298 / 99$, Art. $4^{\circ} \S 3^{\circ}$, cegos e baixa visão. Em alguns se pode observar, inclusive, que pessoas com visão, com os olhos vendados, participaram dos estudos de usabilidade da tecnologia, devido ao fato da dificuldade em encontrar pessoas com deficiências visuais disponíveis para participar deste estudo, conforme constatado na questão de pesquisa 1 .

Contudo, é importante considerar, cuidadosamente, se os participantes com visão vendada são uma amostra razoável ao generalizar para participantes cegos, como alerta Giudice et al. (2012). Mesmo que alguns estudos com gráficos auditivos e mapas táteis 
não tenham encontrado diferenças significativas entre participantes deficientes visuais e videntes com os olhos vendados. Giudice ainda ressalta que o desempenho dos participantes com visão vendada nos experimentos representa uma estimativa conservadora da eficácia da interface, já que, provavelmente, este grupo esteja menos acostumado a usar as dicas hápticas como um modo primário de coleta de informações. Entretanto, os modos de exploração de um gráfico (com as duas mãos pelos deficientes visuais) também são diferentes do que pode lidar a erro no design da ferramenta.

Dos estudos apreciados por este trabalho, apenas 36 citaram as características de deficiência visual dos participantes, onde: 179 participantes eram cegos (41\%), 84 baixa visão (19\%) e 171 não tinham deficiência visual (40\%). Outros 5 estudos não citam o nível de deficiência dos participantes, somando 165 participantes.

Para responder à questão 3: Para quais os níveis de escolaridade estão sendo destinados os estudos? As pesquisas desenvolvidas englobam teste com alunos deficientes visuais ou não, com diferentes níveis de escolaridade, desde o ensino primário até pós-graduação. Dos estudos levados em consideração por este trabalho, apenas 10 citaram os níveis de escolaridade dos participantes. Resultando em 28 participantes de nível fundamental $(23 \%), 30$ participantes de nível médio (24\%), 60 participantes em nível de graduação (49\%) e 4 participantes a nível de pós-graduação (4\%).

Para responder à questão 4: Quais estratégias estão sendo usadas por pesquisadores que investigam formas de auxiliar a exploração de gráficos táteis no ensino para deficientes visuais? Nos estudos analisados por esta RSL, encontramos diversas estratégias utilizadas para criação e apresentação de gráficos táteis. Para criação foi possível encontrar 11 soluções sendo 5 que demonstram formas mais tradicionais para tal, utilizando ferramentas computadorizadas para diagramação manual do gráfico tátil. Outros 6 estudos utilizaram ferramentas que automatizam, ou pelo menos, sem automatizam o processo de diagramação do gráfico tátil fazendo com que esta tarefa, trabalhosa e demorada, seja executa em alguns minutos.

Para apresentação de gráficos táteis, foi possível identificar 44 soluções com diferentes estratégias que podem substituir as respostas sensoriais visuais, ausentes ou falhos em alunos com DV, por respostas sensoriais auditivas e hápticas. Os resultados estão divididos em: 9 soluções (21\%) retornam um feedback de áudio para auxiliar os DVs a acessar informações gráficas; 14 soluções (32\%) fornecem um feedback háptico tátil por meio de vibração, temperatura, eletro vibração ou até mesmo por certo nível de dor; 1 solução ( $2 \%$ ) empregam estratégias de feedback háptico cinestésicos causando um sentido de força e movimento aplicado aos deficientes visuais para acessar as informações gráficas. Por último, 20 soluções (45\%) fornecem um feedback híbrido, combinando dois ou três formas de feedback para aumentar o nível de acessibilidade e respostas sensoriais.

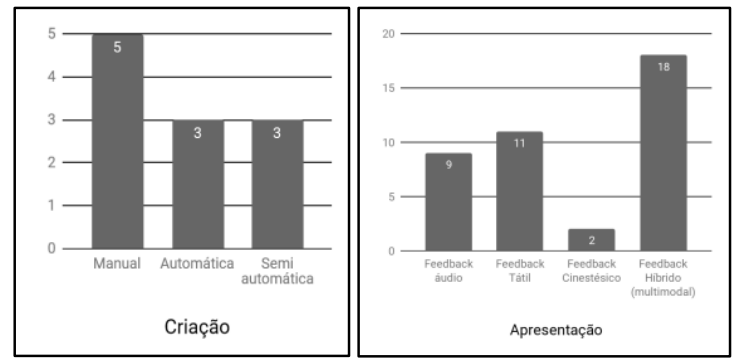

Figura 4. À esquerda, gráfico com as estratégias para criação de conteúdos gráficos. À direita, gráfico com as estratégias de apresentação dos conteúdos gráficos. 
Para responder à questão 5: Os estudos reportados indicam que o uso da tecnologia assistiva desenvolvida é eficaz para auxiliar na leitura e interpretação de gráficos táteis por alunos com deficiência visual? Como é esperado de toda e qualquer tecnologia assistiva que tenha o objetivo facilitar o desenvolvimento de atividades da vida diária por pessoas com deficiência [Pupo; Melo; Ferrés, 2006], da mesma forma para tecnologias assistivas voltadas para nossa problemática também é esperado que consigam facilitar na exploração de gráficos táteis e foi o observado nos estudos analisados por esta RSL.

Para responder à questão 6: Para quais tipos de conteúdo gráficos a solução foi aplicada? Nos estudos analisados por esta RSL encontramos soluções para diversos tipos de conteúdo. Para as ferramentas de criação pudemos verificar que: 6 soluções foram aplicadas à gráficos matemáticos; 5 a desenhos variados. Já no que diz respeito às soluções para apresentação de conteúdos gráficos: 14 foram aplicadas aos conteúdos de gráficos matemáticos; 2 aos objetos 3D; 4 às formas geométricas simples; 8 aos mapas e plantas baixa, utilizando para orientação do deficiente visual em um determinado espaço; e 16 soluções aplicadas aos diversos tipos de conteúdo gráficos, não se restringindo a apenas uma aplicabilidade.

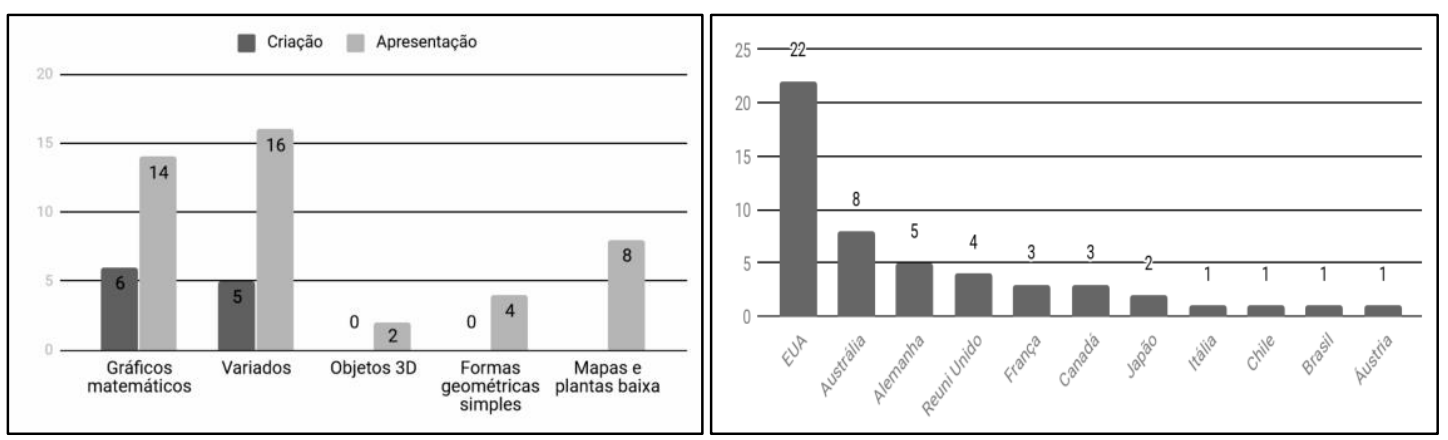

Figura 5. À esquerda, gráfico representando o tipo de conteúdo para a quais as soluções foram aplicadas. À direita, gráfico da distribuição geográfica dos estudos desta RSL.

Para responder à questão 7: Em quais países estão sendo realizados os estudos? A RSL demonstrou um alto número de estudos voltado para esta área, embora poucos tenham sido realizados em países em desenvolvimento, onde o investimento para este tipo de pesquisa é aquém do ideal. Acreditamos que este seja uma das possíveis justificativas para esta RSL ter retornado este resultado. Isto corrobora com a necessidade do desenvolvimento de estudos para melhorar a acessibilidade e inclusão dos deficientes visuais em países em desenvolvimento, mesmo no Brasil, e assim poder elevar o grau de inclusão desta parcela da sociedade, muitas vezes, deixada de lado.

\section{Conclusões}

Essa revisão foi realizada durante os meses de dezembro de 2017 e março de 2018, com atualização realizada nos meses de janeiro e fevereiro de 2019. Para a realização da pesquisa foi definido um protocolo com as atividades de planejamento e condução da revisão. Com os critérios definidos no protocolo foi possível responder às questões definidas, obter uma visão geral das tecnologias assistivas além de identificar oportunidades futuras de pesquisas. As principais conclusões deste trabalho são:

1 - Não foram encontradas tecnologias concretas que possibilitam aos deficientes visuais elaborar conteúdos gráficos, sendo esse um campo que oferece oportunidades para novas pesquisas; 
2 - É preciso levar em consideração um design participativo e centrado no usuário, pois, quando se tem, dentro do projeto participantes com a deficiência alvo do problema, é muito mais provável que todos os detalhes necessários para o sucesso desta solução sejam alcançados. Giudice et al. (2012) acreditam que "esta abordagem evita uma armadilha de engenharia, onde desenvolvimento é impulsionado pela eficiência computacional e suposições dos projetistas, muitas vezes errôneas, sem contar com o feedback do usuário final". Da mesma forma, isto justifica, também, a necessidade de certa cautela na inclusão de videntes vendados nos testes de usabilidade já que estes não possuem características inerentes às pessoas com deficiência visual e os resultados dos testes podem ser um falso positivo.

3 - Ferramentas com feedbacks híbridos (multimodais) são as mais bem aceitas pelos deficientes visuais por permitir uma melhor compreensão dos conteúdos já que são exploradas várias formas de feedbacks ao mesmo tempo. Assim novas tecnologias assistivas podem usar este recurso para obter maior eficácia.

4 - Os estudos indicam que há diversos recursos e tecnologias sendo produzidos e disponibilizados à população, o que reporta a necessidade de que mais profissionais se apropriem destas tecnologias que pode impactar diretamente a autonomia de pessoas com deficiência visual. Contudo, é de extrema importância que o desenvolvimento destas tecnologias assistivas considere os feedbacks dos principais usuários potenciais sobre a deficiência e funcionalidade dos mesmos na vida diária como discutido.

5 - Por fim, ressalta-se que, a maioria dos estudos encontrados, foram realizados em países desenvolvidos, uma vez que, através dos procedimentos de busca adotados, foi reportado apenas um trabalho nacional (e mais um outro trabalho para o resto da América Latina). Cabe-nos mencionar que uma das prováveis razões para essa lacuna no país seja a dificuldade que pesquisadores brasileiros tenham com relação ao financiamento de pesquisas dessa natureza que potencialmente envolvem equipamentos e tecnologias de alto custo. Desta forma é possível pensar em alternativas para reduzir o custo no desenvolvimento de tecnologias assistivas que utilizam dispositivos e hardwares comuns e de fácil acesso. Outra provável razão é que, pela ausência de ferramentas que auxiliam o deficiente visual durante o seu currículo escolar, há uma grande evasão escolar e poucos alunos deficientes visuais alcançam o ensino superior, menos ainda nas áreas de engenharia. As problemáticas encontradas pelos deficientes visuais permanecem, em consequência, longe da percepção dos pesquisadores. Nota-se que esta pesquisa é inclusive, em grande parte, motivada pela presença de um aluno deficiente visual em um curso de engenharia do campus. Desta forma, teria necessidade de romper um ciclo vicioso: há poucos alunos deficientes visuais em cursos superiores de engenharia pela ausência de tecnologias assistivas, há poucas tecnologias assistivas pela raridade dos deficientes visuais nos cursos superiores em engenharia.

Apesar das diretrizes para uma RSL terem sido seguidas é oportuno salientar que, este trabalho, possui algumas limitações que criam oportunidades para próximos trabalhos devido ao fato de que alguns artigos relevantes podem ter ficado fora do estudo por não estarem disponíveis, de forma gratuita, além da quantidade de fontes utilizadas, que podem ser ampliadas para uma maior abrangência e cobertura do estudo, incluindo fontes para pesquisas manuais, mesmo que tenha sido realizada pesquisa no portal de periódico da CAPES que indexa muitas outras bases bibliográficas.

\section{Referências}

Baker, Catherine M et al. Tactile Graphics with a Voice. ACM Transactions on Accessible 
Computing, v. 8, n. 1, p. 1-22, 2016.

Bau, Olivier et al. TeslaTouch: Electrovibration for Touch Surfaces. In: Proceedings of the 23nd annual ACM Symposium on user interface software and technology - UIST '10 2010, Anais. New York, USA: ACM Press, 2010. Disponível em: $<$ http://dl.acm.org/citation.cfm?id=1866074\%5Cnpapers://c80d98e4-9a96-44878d06-8e1acc780d86/Paper/p12800>

Bornschein, Jens; Bornschein, Denise; Weber, Gerhard. (2018) Blind Pictionary: Drawing Application for Blind Users. In: 2018 CHI Conf. Human Factors Comput. Systems. New York, NY, USA.

Brasil. Subsecretaria Nacional de Promoção dos Direitos da Pessoa com Deficiência. Comitê de Ajudas Técnicas. Tec. Assistiva (CAT). - Brasília: CORDE, 2009. 138 p.

Brown, Craig; Hurst, Amy. (2012) Viztouch: Automatically Generated Tactile Visualizations of Coordinate Spaces. Proceedings of the Sixth International Conference on Tangible, Embedded and Embodied Interaction - TEI '12.

Costa, Laisa C. P. Et al. (2015) Accessible educational digital book on tablets for people with visual impairment. IEEE Trans. Consum. Electron., v. 61, n. 3, p. 271-278.

Fusco, Giovanni; Morash, Valerie S. (2015) The Tactile Graphics Helper: Providing Audio Clarification for Tactile Graphics Using Machine Vision. In: Proc. 17TH Int. ACM SIGACCESS Conf. Comput. \& Access. New York, NY, USA.

Gardner, John; Bulatov, Vladimir. (2006) Scientific Diagrams Made Easy with IVEO. ICCHP - Inter. Conf. On Computers Helping People with Special Needs.

Giudice, Nicholas A. Et al. (2012) Learning Non-visual Graphical Information Using a Touch-based Vibro-audio Interface. In: Proc. Of $14^{\text {th }}$ International ACM Conference on Computers and Accessibility 2012, New York, NY, USA.

Goncu, Cagatay; Marriott, Kim. (2008) Tactile Chart Generation Tool. In: Proc. Of the $10^{\text {th }}$ International Conference on Computer and Accessibility 2008, New York, USA.

Goncu, Cagatay; Marriott, Kim. (2011) Gravvitas: Generic Multi-touch Presentation of Accessible Graphics. In: Proc. of the $13^{\text {th }}$ IFIP International Conference on HumanComputer Interaction - Vol Part I 2011, Berlin, Heidelberg. Springer-Verlag.

Goncu, C.; Marriott, K. Creating ebooks with Accessible Graphics Content. In: Proc. of the 2015 ACM Symposium on Document Engineering. 2015a, New York, NY, USA.

Goncu, Cagatay; Marriott, Kim. Gracalc: An Accessible Graphing Calculator. In: Proc. of the $17^{\text {th }}$ Intern. Conf. on Computer and Accessibility. 2015b, New York, USA.

Goncu, Cagatay; Marriott, Kim; Hurst, John. (2010) Usability of Accessible Bar Charts. In: Proc. of the $6^{\text {th }}$ International Conference on Diagrammatic Representation and Inference 2010, Berlin, Heidelberg.

He, Liang et al. (2017) Tactile: A Preliminary Toolchain for Creating Accessible Graphics with 3D-Printed Overlays and Auditory Annotations. In: Proc of the $19^{\text {th }}$ Conference on Computers and Accessibility 2017, New York, NY, USA.

Hribar, Victoria E.; Deal, Laura G.; Pawluk, Dianne T. V. Displaying braille and graphics with a "tactile mouse". In: Proceedings of the $14^{\text {th }}$ International ACM SIGACCESS Conference On Computers And Accessibility - ASSETS '12 2012, New York, New York, USA. $2012 . \quad$ Anais. Disponível em: 
$<$ http://doi.acm.org/10.1145/2384916.2384978>

Jayant, Chandrika et al. (2007) Automated tactile graphics translation. Proc. Of the 9th intern. ACM SIGACCESS conf. On Computers and accessibility - Assets 07.

Kitchenham, Barbara; Charters, Stuart. Guidelines for performing Systematic Literature Reviews in Software Engineering Version 2.3. EBSE Techinal Report. Keele, UK: Keele University, 2007.

Krufka, S. E.; Barner, K. E. (2005) Automatic production of tactile graphics from scalable vector graphics. In: ASSETS 2005 - 7th International ACM SIGACCESS Conference on Computers and Accessibility 2005.

Landau, Steven et al. (2008) Demonstration of a universally accessible audio-haptic transit map built on a digital pen-based platform. 3rd International Haptic and Auditory Interaction Design Workshop.

Landau, Steven; Russel, Michael; Erin, Jane N. (2007) Using the Talking Tactile Tablet as a Testing Accommodation. Review: Rehabilitation and Educ. for Blindness and Visual Impairment.

Maucher, Thorsten; Schemmel, Johannes; Meier, Karlheinz. (2000) The Heidelberg Tactile Vision Substitution System. International Conference on Computers Helping People with Special Needs (ICCHP2000).

Mcgookin, David K.; Brewster, Stephen a. (2006) Multivis: improving access to visualisations for visually impaired people. Proc. of ACM CHI 2006 Conference on Human Factors in Computing Systems.

Mcgookin, David; Brewster, Stephen; Jiang, Weiwei. Investigating touchscreen accessibility for people with visual impairments. In: Proceedings of the 5th nordic conference on human-computer interaction building bridges, New York: USA, 2008.

Petit, Grégory et al. Refreshable Tactile Graphics Applied to Schoolbook Illustrations for Students with Visual Impairment. In: Proceedings of the $10^{\text {th }}$ International ACM SIGACCESS Conference on Computers and Accessibility 2008, New York, NY, USA. Anais... New York, NY, USA: ACM, 2008. Disponível em: $<\mathrm{http}$ ://oi.acm.org/10.1145/1414471.1414489>

Pupo, Deise T.; Melo, Amanda Meincke; Ferrés, Sofia Pérez. Acessibilidade: Discurso e Prática no Cotidiano das Bibliotecas. Campinas - SP: UNICAMP/Biblioteca. 2006.

Silva, Chirley C. M. da; Turatto, Jaqueline; Machado, Lizete Helena. Os deficientes visuais e o acesso à informação Rev. Biblioteconomia em Santa Catarina, 2002.

Suzuki, Ryo et al. Fluxmarker: Enhancing Tactile Graphics with Dynamic Tactile Markers. In: Proc. of the 19th International Conference on Computers and Accessibility 2017, New York, USA.

Yu, Wai; Brewster, Stephen. (2002) Comparing two haptic interfaces for multimodal graph rendering. Proceedings - 10th Symposium on Haptic Interfaces for Virtual Environment and Teleoperator Systems, HAPTICS 2002, p. 3-9.

Zebehazy, Kim T.; Wilton, Adam P. (2014) Straight from the Source: Perceptions of Students with Visual Impair. about Graphic Use. Journal of Visual Impair. \& Blindness. 